\title{
Coherent Ratchets in Driven Bose-Einstein Condensates
}

\begin{abstract}
C. E. Creffield and F. Sols
Departamento de Física de Materiales, Universidad Complutense de Madrid, E-28040, Madrid, Spain (Received 5 August 2009; revised manuscript received 29 October 2009; published 12 November 2009)

We study the response of a Bose-Einstein condensate to an unbiased periodic driving potential. By controlling the space and time symmetries of the driving we show how a directed current can be induced, producing a coherent quantum ratchet. Weak driving induces a regular behavior, and space and time symmetries must both be broken to produce a current. For strong driving, the behavior becomes chaotic and the resulting effective irreversibility means that it is unnecessary to explicitly break time symmetry. Spatial asymmetry alone is then sufficient to produce the ratchet effect, even in the absence of interactions, and although the system remains completely coherent.
\end{abstract}

DOI: 10.1103/PhysRevLett.103.200601

Introduction.-The physics of ratchets, systems that exhibit directed motion in the absence of an external bias, has undergone extremely rapid development in recent years $[1,2]$. The concept is very general, and ranges from new technological forms of manipulating and directing matter at nanoscale levels, to understanding how systems in nature such as biological molecular motors function. Fundamentally a ratchet must satisfy two essential requirements: the system must be driven out of equilibrium by an external force, and the relevant space and time symmetries, which would otherwise forbid the generation of directed currents, must be broken.

A well-known example is provided by a Brownian particle in a periodic potential. Driving the system from equilibrium by either pulsing the potential ("flashing ratchet") or tilting it ("rocking ratchet") produces a current if the spatial or temporal symmetry of the driving force is broken. In the most commonly studied overdamped regime, the ratchet current arises from the rectification of random fluctuations, and accordingly noise and dissipation are essential ingredients. This is not true in general, however, and surprisingly it has been shown recently [3-9] that ratchet effects can also occur even in completely coherent systems.

Considerable progress in this direction has been made by considering the quantum kicked rotor. Experimentally this system can be realized extremely well in gases of ultracold atoms held in pulsed optical lattices. While it was originally thought that ratchet effects would only arise in systems with an underlying mixed classical phase space [3], recent work has shown that they can also arise when the phase space is globally chaotic. In Ref. [4] a quantum Hamiltonian ratchet of this type was studied both theoretically and experimentally, in which the current arose from the generation of an asymmetry in the momentum distribution due to the desymmetrization of the system's Floquet states. An alternative scheme, developed in the context of quantum maps [5], is to use interference effects to produce an imbalance in the phase-space distribution. Quantum
PACS numbers: 05.60.Gg, 03.75.Kk, 05.45.-a, 67.85.Hj

resonances, where the period of the kicks is matched to the inverse recoil velocity of the optical lattice, have also been proposed $[6,7]$ as a means of producing ratchet accelerators.

In this Letter we consider an optically trapped BoseEinstein condensate (BEC), since the macroscopically protected coherence and excellent controllability of these systems make them ideal subjects for investigating quantum transport effects. Instead of kicking the system $[8,10]$, we use a smoothly varying potential and so can expect to produce less heating effects, which we verify explicitly by evaluating the fraction of noncondensed atoms. By choosing a form for the driving which enables us to separately break space and time symmetries, we find that we can induce a directed current in a BEC starting from a symmetric initial state. This occurs by two distinct mechanisms; for weak driving, the system undergoes regular oscillations, and both space and time symmetries in the driving must be broken. Conversely, for strong driving, the system's dynamics becomes chaotic, and this produces an effective irreversibility which means it is not necessary to explicitly break the time symmetry.

Model. - We consider a BEC confined in a toroidal trap [11] with a cross section much smaller than the trap's radius, $R$. The system can thus be described by an effective one-dimensional Gross-Pitaevskii equation (GPE)

$$
H(t)=-\frac{1}{2} \frac{\partial^{2}}{\partial x^{2}}+g|\psi(x, t)|^{2}+K V(x, t),
$$

where $x$ parametrizes distance around the trap, and we measure all energies in units of $\hbar^{2} / 2 m R^{2}$. The short-range interactions between the atoms in the condensate are described by a mean-field term with strength $g$, and the condensate is driven by a time-periodic external potential with zero mean by modulating the amplitude of the optical potential. The archetypal form of a ratchet potential [2] is $V=\sin (x)+\alpha \sin (2 x+\phi)$, where $V$ is symmetric for $\phi=\pi / 2$, and is maximally asymmetric for $\phi=0, \pi$. We make the unusual choice of factoring the potential 
into separate spatial and time-dependent components of this form $V(x, t)=V(x) f(t)$

$$
\begin{gathered}
V(x)=\sin (x)+\alpha \sin (2 x), \\
f(t)=\sin (\omega t)+\beta \sin (2 \omega t) .
\end{gathered}
$$

It is important to note that this potential does not correspond to either a purely rocking or flashing ratchet, and has the appealing feature of allowing the space and time symmetries of the system to be controlled independently. We plot the form of $V(x)$ in the inset of Fig. 1(a), and show that for nonzero values of $\alpha$ the potential becomes skewed, breaking both inversion symmetry and shift symmetry. A static spatial potential of this type has been recently studied experimentally in [12].

Results.-To probe the behavior of the system we evaluate $I(t)=\langle\psi(x, t)|p| \psi(x, t)\rangle$ as a measure of the current flowing in the ring. As an initial condition, we choose the zero-current state $\psi(x, 0)=1 / \sqrt{2 \pi}$, which is convenient for experiment as it is the ground state of the undriven Hamiltonian, and so can be prepared by cooling. We numerically integrate the wave function in time using a split-operator method, and henceforth we shall take the driving frequency $\omega=1$. In Fig. 1(a) we show the timeaveraged current, $\bar{I}$, obtained by integrating the system over 100 driving periods. For weak driving we can note a sharp peak centered at $K=0.15$. As $K$ is increased from this value the current drops, becomes negative, and passes through a negative peak at $K=2.4$ This second peak is associated with an enhancement of (negative) current over a rather broad range of driving amplitudes.

We show the time dependence of the current for weak driving in Fig. 1(b). It is clear that the sharp peak in $\bar{I}$ is associated with regular oscillations of the current. For $g=$ 0 this oscillation is sinusoidal with a period much larger than $T=2 \pi / \omega$. Increasing $g$ initially slightly enhances the amplitude of the oscillation, while also deforming its waveform. As $g$ is increased further, however, the oscillation's amplitude becomes abruptly suppressed [see Fig. 2(d)]. Examining the evolution of the system in detail reveals that the oscillation occurs chiefly between the states $|0\rangle$ and $|2\rangle$, where $|n\rangle$ denotes an eigenstate of the undriven Hamiltonian with (quantized) momentum $n \hbar$. While a symmetric driving would equally populate states with positive and negative momentum, producing zero current, interference effects cause the asymmetric form to preferentially drive the system to $|+2\rangle$ rather than $|-2\rangle$, thus inducing a net current. A perturbative study of the Floquet states explains this result [13].

To qualitatively study this phenomenon, let us truncate the wave function to just the two components of interest, $\psi(x, t)=A+B \exp (2 i x)$, where $|A|^{2}+|B|^{2}=1 / \sqrt{2 \pi}$. Under the action of the Hamiltonian [Eq. (1)], this yields the equation of motion for the expansion coefficients $\chi=(A, B)$

$$
i \dot{\chi}=\left[-\frac{\alpha K}{2} f(t) \sigma_{y}-\left(1+\frac{g}{2}\left[|A|^{2}-|B|^{2}\right]\right) \sigma_{z}\right] \chi,
$$

where $\sigma_{j}$ are the Pauli matrices. We may now visualize the time evolution of the system using the Bloch sphere representation, where the north/south poles correspond to occupation of the states $|0\rangle /|2\rangle$. For $g=0$ the Bloch vector will evolve under the influence of a fictitious magnetic field $B_{y}=\alpha K f(t)$, and so will simply make a Larmor orbit in the $x-z$ plane as shown in Fig. 2(a). This corresponds to the sinusoidal oscillation displayed in Fig. 1(a). For a larger value of $g$ the Bloch vector will execute a more complicated "figure-of-eight" motion under the combined influence of $B_{x}$ and $B_{z}$, shown in Fig. 2(b), producing the nonsinusoidal current oscillations seen in Fig. 1(b). When $g$ is increased further, the magnitude of the fictitious field component $B_{z}$ is enhanced, until the Bloch vector is confined to making rapid circular orbits [Fig. 2(c)] near the north pole, in a process directly analogous to the nonlinear phenomenon termed self-trapping [14]. These small orbits correspond to the low amplitude, high frequency oscillations seen in Fig. 1(b) for large values of $g$. In Fig. 2(d) we show in detail how this transition occurs by plotting the amplitude of the current oscillations as $g$ is increased, and demonstrate that this simple model indeed captures the main features of this effect.
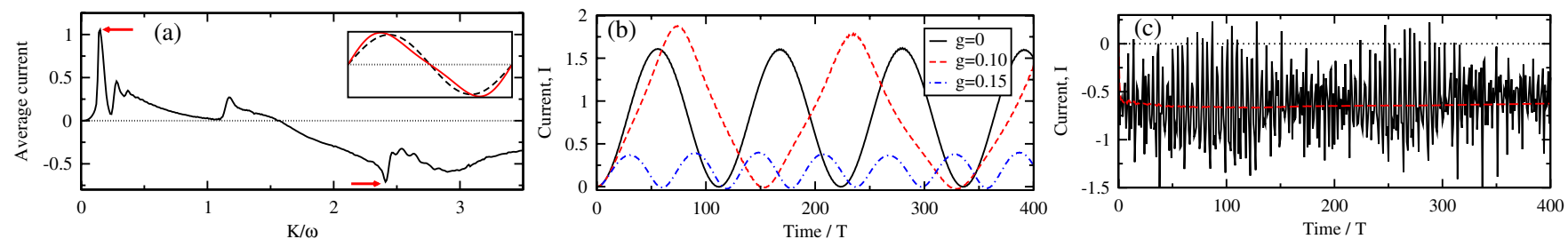

FIG. 1 (color online). (a) Time-averaged current (averaged over 100 periods), a function of driving strength $K$ for symmetry parameters $\alpha=\beta=0.2$ and nonlinearity $g=0.1$. Clear peaks at $K=0.15$ and $K=2.4$ are marked with arrows. Inset: Plot of the driving potential $V(x)$. For $\alpha=0$ (black dashed curve) it is symmetric, but for $\alpha=0.2$ (solid red curve) it has an asymmetric sawtooth form. (b) Current induced for weak driving $(K=0.15)$. For $g=0$ the current exhibits a smooth sinusoidal oscillation; as $g$ is increased the oscillations are initially enhanced in amplitude and deviate from sinusoidal form, and subsequently become highly suppressed. (c) Current induced for strong driving $(K=2.4, g=0.1)$. In contrast to the previous case the current shows rapid quasiperiodic oscillations, which nonetheless maintain a stable time-averaged value (red dashed line). 

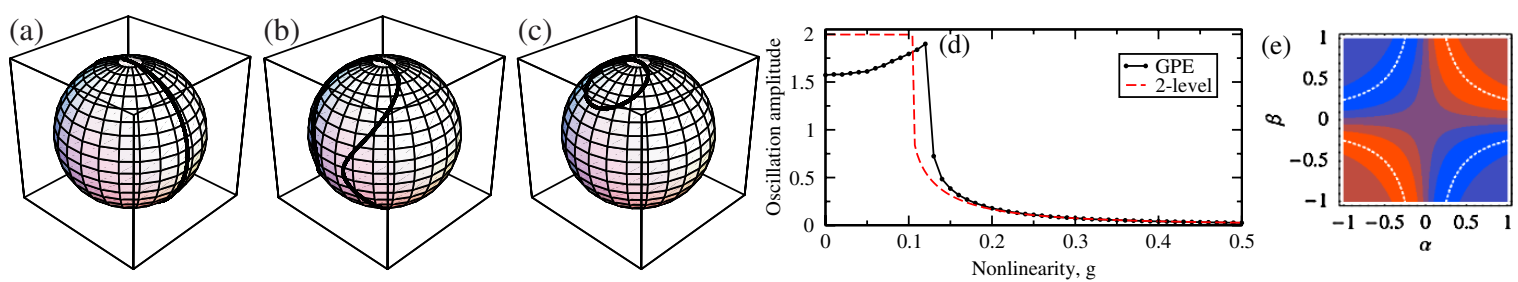

FIG. 2 (color online). (a)-(c) Bloch sphere representation of the evolution of the effective two-level model [Eq. (4)] for weak-driving ( $K=0.15$ ). (a) $g=0$ : The Bloch vector makes a circular orbit in the $x-z$ plane, corresponding to a sinusoidal current oscillation. (b) $g=0.1$ : The Bloch vector makes a more complicated "figure-of-eight" orbit, producing the distortion from the sinusoidal waveform. (c) $g=0.15$ : A phenomenon analogous to self-trapping occurs, and the Bloch vector is confined to small orbits in the vicinity of the north pole, implying an interaction-induced suppression of current. (d) The suppression of the current occurs at a very precisely defined value of $g$. The two-level approximation results (dashed red line) compare well with the simulation of the full GPE (solid black line). (e) Current produced as a function of the asymmetry parameters $(g=0.10)$. The current vanishes for $\alpha$ or $\beta=0$, and is positive (negative) in the top-left/bottom right (top-right/bottom left) quadrants. Contours of constant current are given by $\alpha \beta=$ cnst. in agreement with the perturbative result [13]; dashed lines show the current maxima.

We now consider the case of strong driving $(K=2.4)$. In this regime the initial state is driven to a larger number of excited levels, and consequently the two-level approximation is no longer valid. The generated current, shown in Fig. 1(c), instead shows an irregular, quasiperiodic character corresponding to the large number of frequency components present. Despite its jagged appearance, the current converges to a stable time average after just a few tens of driving periods. The ratchet current thus arises from a completely different mechanism than before; instead of a regular oscillation between two momentum states, the system now evolves to a superposition of many momentum eigenstates, which crucially has an asymmetric momentum distribution [4]. This produces a dramatic difference between the symmetry dependence of the current in the weak and strong driving regimes. The symmetry properties of this form of driving were analyzed in Ref. [15] for a noninteracting system, and it was concluded that both space and time symmetries needed to be broken to produce a ratchet current. This is indeed the case for weak driving, and in Fig. 2(e) we can clearly see that the current vanishes for $\alpha, \beta=0$. In contrast, when the system is driven strongly only $\alpha \neq 0$ is required, that is, it is sufficient to just break the spatial symmetry, an effect also noted in quantum resonant ratchets [16]. In Fig. 3 we show the current produced for strong driving when the temporal asymmetry $\beta$ is set to zero. Away from $\alpha=0$ we obtain a clear ratchet current whose direction depends on the sign of $\alpha$. This occurs in analogy to the production of a ratchet current in a non-Hamiltonian system [17], but instead of dissipation it is the quasiperiodic evolution of the system which produces the effective irreversibility in time [18]. This contrasts sharply with the results obtained in Ref. [9], where instead the interaction was argued to play the role of breaking the time-reversal symmetry. As a result, the ratchet current we obtain has only very weak dependence on $g$ (as shown in Fig. 3), and occurs even in the noninteracting case.
As well as driving the dynamics of the condensed atoms, the potential also has the effect of exciting atoms out of the BEC to form a thermal cloud. This depletion eventually leads to the destruction of the BEC, and seeing how rapidly this occurs allows us to assess the stability of the BEC under driving [10]. This can be done by making the Bogoliubov approximation, and linearizing the GPE about its ground state. Following the Castin-Dum formalism [19], the mean number of noncondensed atoms at zero temperature is given by $N(t)=\sum_{k \neq 0}\left\langle v_{k}(t) \mid v_{k}(t)\right\rangle$, where $\left(u_{k}, v_{k}\right)$ are the amplitudes of the Bogoliubov quasiparticle operators with quantized momentum $k$. This approximation is valid provided that the number of particles excited from the condensate is small compared to the condensate itself.

In Fig. 4(a) we show the time development of the noncondensed atoms for both weak and strong driving. In all cases the number of atoms does not increase exponentially, but instead follows an approximate power law, $N \propto t^{\gamma}$ with $\gamma \simeq 1.9$, indicating that under the driving the condensate does not exhibit dynamical instability. It is interesting to note that for weak driving the production of noncondensed

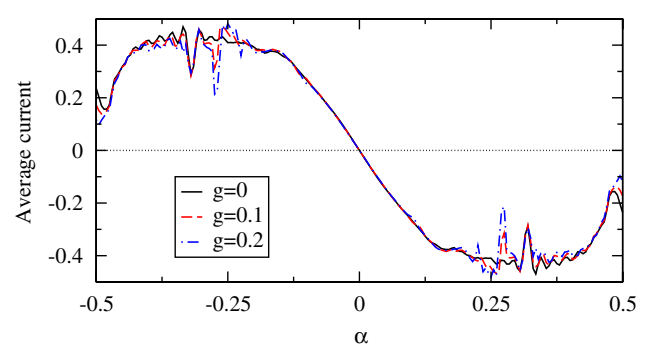

FIG. 3 (color online). Ratchet current produced for strong driving $(K=2.4)$ for a system with no temporal asymmetry (i.e., $\beta=0)$. Nonzero spatial asymmetry $(\alpha \neq 0)$ is required to induce a current, and the direction of the current depends on the sign of $\alpha$. The magnitude of the current depends only weakly on the nonlinearity $g$. 

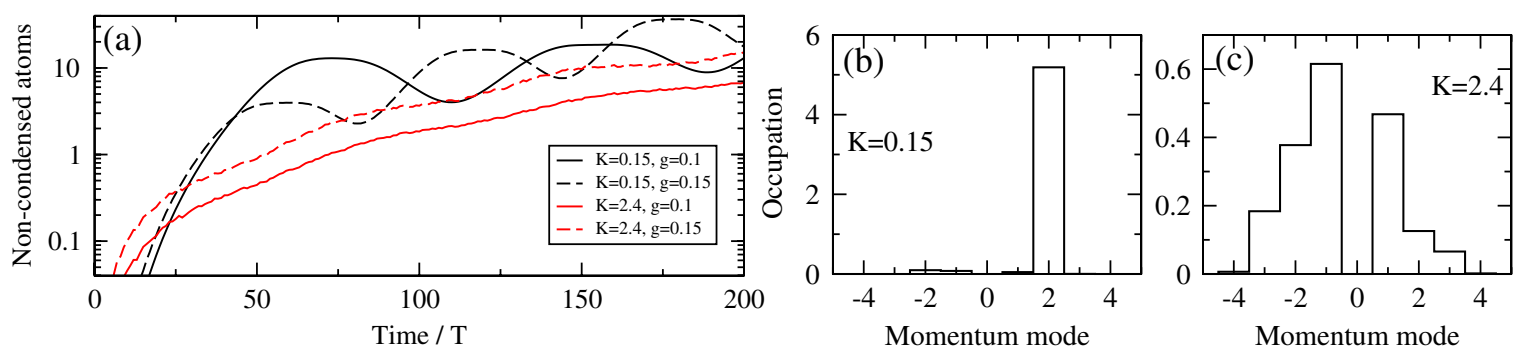

FIG. 4 (color online). (a) For weak driving ( $K=0.15$, upper black curves) the number of noncondensed atoms exhibits significant oscillations, arising from the oscillation of atoms in the condensate. For strong driving $(K=2.4$, lower red curves) the number rises monotonically with a similar approximately power-law rate of increase. (b) Occupation of noncondensed modes after 100 driving periods for weak driving $(g=0.1)$. This distribution mimics the spectrum of the condensate, with a large peak at $n=+2$. (c) As for (b) but for strong driving. The distribution of momentum is again asymmetric, but many modes are populated.

atoms exhibits marked oscillations. These are a consequence of the large, slow current oscillations that the driving generates; the regular sloshing motion periodically produces peaks in the density distribution of the condensate, causing an enhanced emission of particles at those times and locations. As the density distribution can be directly imaged in experiment, this provides a convenient means to study the dynamics of the BEC in this regime. The momentum distribution of the noncondensed atoms also mimics the behavior of the condensate. In Fig. 4(b) we see that weak driving essentially only excites a single Bogoliubov mode, with momentum $k=+2$. As the driving is not resonant with the Bogoliubov frequency, however, this mode does not grow exponentially with time [10] and so dynamical instability is avoided. Conversely, for strong driving a larger number of Bogoliubov modes are excited, and the momentum distribution is strongly asymmetric, again resembling the momentum spectrum of the condensate.

Conclusions. - We have investigated the dynamics of a $\mathrm{BEC}$ under a periodic driving potential. In the weakdriving regime a ratchet current can be generated by breaking both space and time symmetries, and inducing slow, regular oscillations of the condensate. For strong driving a more complicated quasiperiodic dynamics is induced, in which chaos acts to eliminate the long term memory of the system, thus mimicking thermal noise. Accordingly, only the spatial symmetry then needs to be broken to produce a ratchet current. This current should remain stable over time scales comparable to the system's Ehrenfest time, which scales logarithmically with the number of atoms in the condensate [20], as also suggested by numerical results presented in Ref. [9]. For a condensate of $10^{5}$ atoms we estimate this time to be of the order of 50 driving periods. These results expose a new vista of possibilities in manipulating the interplay between the driving potential, interactions and symmetry breaking to induce directed transport in quantum coherent systems.

This work was supported by MICINN (Spain) through Grant No. FIS-2007-65723 and the Ramón y Cajal Program (CEC). The authors thank Ferruccio Renzoni,
Tania Monteiro, and Peter Reimann for stimulating discussions.

[1] P. Reimann, Phys. Rep. 361, 57 (2002).

[2] P. Hänggi and F. Marchesoni, Rev. Mod. Phys. 81, 387 (2009).

[3] H. Schanz, M.-F. Otto, R. Ketzmerick, and T. Dittrich, Phys. Rev. Lett. 87, 070601 (2001).

[4] G. Hur, C. E. Creffield, P. H. Jones, and T. S. Monteiro, Phys. Rev. A 72, 013403 (2005).

[5] L. Ermann, G. G. Carlo, and M. Saraceno, Phys. Rev. E 77, 011126 (2008); 79, 056201 (2009).

[6] J. Gong and P. Brumer, Phys. Rev. Lett. 97, 240602 (2006).

[7] M. Sadgrove et al., Phys. Rev. Lett. 99, 043002 (2007); I. Dana et al., Phys. Rev. Lett. 100, 024103 (2008).

[8] D. Poletti et al., Phys. Rev. A 76, 023421 (2007).

[9] D. Poletti, G. Benenti, G. Casati, P. Hänggi, and B. Li, Phys. Rev. Lett. 102, 130604 (2009).

[10] J. Reslen, C. E. Creffield, and T. S. Monteiro, Phys. Rev. A 77, 043621 (2008).

[11] S. Gupta et al., Phys. Rev. Lett. 95, 143201 (2005); S. E. Olson et al., Phys. Rev. A 76, 061404(R) (2007); C. Ryu et al., Phys. Rev. Lett. 99, 260401 (2007).

[12] T. Salger, C. Geckeler, S. Kling, and M. Weitz, Phys. Rev. Lett. 99, 190405 (2007).

[13] C. E. Creffield and F. Sols (to be published).

[14] T. Anker, M. Albiez, R. Gati, S. Hunsmann, B. Eiermann, A. Trombettoni, and M. K. Oberthaler, Phys. Rev. Lett. 94, 020403 (2005).

[15] S. Denisov, L. Morales-Molina, S. Flach, and P. Hänggi, Phys. Rev. A 75, 063424 (2007).

[16] E. Lundh and M. Wallin, Phys. Rev. Lett. 94, 110603 (2005); D. Poletti, G. G. Carlo, and B. Li, Phys. Rev. E 75, 011102 (2007).

[17] R. Gommers, S. Bergamini, and F. Renzoni, Phys. Rev. Lett. 95, 073003 (2005).

[18] B. Mieck and R. Graham, J. Phys. A 37, L581 (2004).

[19] Y. Castin and R. Dum, Phys. Rev. Lett. 79, 3553 (1997).

[20] J. Martin, B. Georgeot, and D. L. Shepelyansky, Phys. Rev. Lett. 101, 074102 (2008). 